\title{
Analytical Solution for the Time Fractional BBM-Burger Equation by Using Modified Residual Power Series Method
}

\author{
Jianke Zhang $\mathbb{D}^{1,2}$ Zhirou Wei, $^{1}$ Longquan Yong, $^{3}$ and Yuelei Xiao ${ }^{4}$ \\ ${ }^{1}$ School of Science, Xi'an University of Posts and Telecommunications, Xi'an 710121, China \\ ${ }^{2}$ Shaanxi Key Laboratory of Network Data Analysis and Intelligent Processing, Xi'an University of Posts and Telecommunications, \\ Xi'an, Shaanxi 710121, China \\ ${ }^{3}$ School of Mathematics and Computer Science, Shaanxi University of Technology, Hanzhong 723000, China \\ ${ }^{4}$ Institute of IOT and IT-based Industrialization, Xi'an University of Posts and Telecommunications, Xi'an 710061, China \\ Correspondence should be addressed to Jianke Zhang; jiankezh@163.com
}

Received 24 February 2018; Revised 3 May 2018; Accepted 1 August 2018; Published 3 October 2018

Academic Editor: Lucia Valentina Gambuzza

Copyright (C) 2018 Jianke Zhang et al. This is an open access article distributed under the Creative Commons Attribution License, which permits unrestricted use, distribution, and reproduction in any medium, provided the original work is properly cited.

\begin{abstract}
In this study, a generalized Taylor series formula together with residual error function, which is named the residual power series method (RPSM), is used for finding the series solution of the time fractional Benjamin-Bona-Mahony-Burger (BBM-Burger) equation. The BBM-Burger equation is useful in describing approximately the unidirectional propagation of long waves in certain nonlinear dispersive systems. The numerical solution of the BBM-Burger equation is calculated by Maple. The numerical results show that the RPSM is reliable and powerful in solving the numerical solutions of the BBM-Burger equation compared with the exact solutions as well as the solutions obtained by homotopy analysis transform method through different graphical representations and tables.
\end{abstract}

\section{Introduction}

Today, fractional differential equations are more and more important in many fields, such as mathematics and dynamic systems $[1,2]$. The persons who firstly proposed fractional differential equations were Leibniz and L'Hopital in 1695. Lakshmikantham and Vatsala [3] discussed the basic theory for the initial value problem involving Riemann-Liouville differential operators by fractional differential equations. Diethelm and Ford [4] proposed the analytical questions of existence and uniqueness of solutions by fractional differential equations. And many other academics studied different theories in fractional differential equations. However, most of the problems do not possess analytical solution, and thus, a lot of numerical methods have been developed to solve these fractional differential equations.

Many different methods are introduced to develop an approximate analytical solution for the fractional differential equations and systems, such as the variational iteration method [5], homotopy analysis transform [6], homotopy asymptotic method [7], $\left(G^{\prime} / G\right)$ expansion method [8], polynomial least squares method [9], and finite difference method [10]. Recently, an analytical method based on power series expansion without linearization, discretization, or perturbation has been introduced and successfully applied to many kinds of fractional differential equations arising in strongly nonlinear and dynamic problems. The method was named residual power series method (RPSM) [11-27], which was used to find the analytical solution for several classes of time fractional differential equations. The residual power series method has been widely used in different fields. In [11], some important theorems that are related to the classical power series have been generalized to the fractional power series by El-Ajou et al. These theorems are constructed by using Caputo fractional derivatives. They also presented and discussed the explicit and approximate solutions of the nonlinear fractional KdV-Burgers equation with timespace-fractional derivatives in [12]. Moaddy et al. [13] proposed that the residual power series method can be applied to differential algebraic equation systems. Jaradat et al. [14] 
solved the time fractional Drinfeld-Sokolov-Wilson system by residual power series method. In [15-21], residual power series method, as a powerful method, was used to solve the other time fractional differential equations. Residual power series method was also used for the time fractional Gardner [23] and Kawahara equations in [22], the time fractional Phi-4 equation in [24], the fractional population diffusion model [25], the generalized Burger-Huxley equation [26], and the time fractional two-component evolutionary system of order 2 [27].

In this paper, an analytical solution of the time fractional Benjamin-Bona-Mahony-Burger equation (called BBMBurger equation) is proposed by residual power series method. The BBM-Burger equation describes the mathematical model of propagation of small-amplitude long waves in nonlinear dispersive media. It is well known that the BBM equation is a refinement of the $\mathrm{KdV}$ equation. The BBMBurger equation and the $\mathrm{KdV}$ equation are relevant to the wave breaking models [28]. The KdV equation came from water waves, and the KdV equation as a model was used for long waves in many other physical systems. But in some physical system of long waves, the KdV equation was not applicable. So, the BBM-Burger was proposed; it described unidirectional propagation of long waves in a certain nonlinear dispersive system [28-30]. The integer order of the BBMBurger equation can be written as

$$
u_{t}-u_{x x t}-\alpha u_{x x}+u u_{x}+\beta u_{x}=0, \quad x \in\left[x_{L}, x_{R}\right],
$$

where $\alpha$ and $\beta$ are positive constants and $x \in\left[x_{L}, x_{R}\right]$ is a domain partition.

In order to discuss the dynamic physical system, the time fractional BBM-Burger equation was proposed. The BBMBurger equation can be written in time fractional operator form as [31]

$$
D_{t}^{\alpha} u-u_{x x t}+u_{x}+\left(\frac{u^{2}}{2}\right)_{x}=0, \quad t>0, x \in I \subseteq R, \alpha \in(0,1],
$$

where $\alpha$ is a parameter, which is the order of the time fractional derivative and is located in the range of $(0,1]$. The initial condition is

$$
u(x, 0)=\operatorname{sech}^{2}\left(\frac{x}{4}\right)
$$

If $\alpha=1$, the exact solution [32] is

$$
u(x, t)=\operatorname{sech}^{2}\left(\frac{x}{4}-\frac{t}{4}\right) .
$$

The rest of the paper is as follows. In Section 2, some basic definitions about the Caputo and modified residual power series method are introduced. In Section 3, we use residual power series method to solve the time fractional BBM-Burger equation specifically. Numerical results and discussions are presented by graphics and charts in Section 4. At last, the conclusion was drawn in Section 5.

\section{Modified Residual Power Series Method}

In this section, the definition of the Caputo fractional is introduced systematically. And this section also presents the most details of the modified residual power series method. Fractional residual power series method is used to solve many kinds of differential equations, and this method is effective in calculating these equations.

Definition 1 (see [33]). Let $f(t):[0,+\infty) \rightarrow R$ be a function and $n$ be the upper positive integer of $\alpha(\alpha>0)$. The Caputo fractional derivative is defined by

$$
D^{\alpha} f(t)=\left\{\begin{array}{l}
\frac{1}{\Gamma(n-\alpha)} \int_{0}^{x}(t-\tau)^{n-\alpha-1} \frac{d^{n} f(\tau)}{d \tau^{n}} d \tau, \quad n-1<\alpha<n, \\
\frac{d^{n} f(x)}{d x^{n}}, \quad \alpha=n \in N .
\end{array}\right.
$$

Theorem 1 (see [33]). The Caputo fractional derivative of the power function satisfies

$$
D^{\alpha} x^{q}=\left\{\begin{array}{l}
\frac{\Gamma(q+1)}{\Gamma(q+1-\alpha)} x^{q-\alpha}, \quad \alpha \leq q, \\
0, \quad \alpha>q .
\end{array}\right.
$$

Below, we introduce some definitions and theorems related to the fractional power series used in this paper. These important theorems that are related to the fractional power series were presented by El-Ajou et al. [11, 12]. These theorems are constructed by using Caputo fractional derivatives.

Definition 2 (see $[11,12])$. A power series expansion of the form

$$
\sum_{m=0}^{\infty} c_{m}\left(t-t_{0}\right)^{m \alpha}=c_{0}+c_{1}\left(t-t_{0}\right)^{\alpha}+c_{2}\left(t-t_{0}\right)^{2 \alpha}+, \ldots,
$$

for $0 \leq n-1<\alpha \leq n$ and $t \geq t_{0}$, is called fractional power series about $t=t_{0}$, where $t$ is a variable and $c_{m}$ are constants called the coefficients of the series.

Theorem 2 (see [11]). Suppose that $f$ has a fractional power series representation at $t=t_{0}$ of the form

$$
f(t)=\sum_{m=0}^{\infty} c_{m}\left(t-t_{0}\right)^{m \alpha}, \quad 0 \leq n-1<\alpha \leq n, t_{0} \leq t<t_{0}+R .
$$

If $D^{m \alpha} f(t) \in\left(t_{0}, t_{0}+R\right), m=0,1,2, \ldots$, then coefficients $c_{m}$ of (8) are given by the formula 


$$
c_{m}=\frac{D^{m \alpha} f\left(t_{0}\right)}{\Gamma(m \alpha+1)}, \quad m=0,1,2, \ldots,
$$

where $D^{m \alpha}=D^{\alpha} \cdot D^{\alpha} \cdots D^{\alpha}(m-$ times $)$ and $R$ is the radius of convergence.

Definition 3 (see [12]). A power series of the form

$$
\begin{aligned}
\sum_{m=0}^{\infty} f_{m}(x)\left(t-t_{0}\right)^{k \alpha}= & f_{0}(x)+f_{1}(x)\left(t-t_{0}\right)^{\alpha} \\
& +f_{2}(x)\left(t-t_{0}\right)^{2 \alpha}+, \ldots,
\end{aligned}
$$

for $0 \leq n-1<\alpha \leq n$ and $t \geq t_{0}$, is called multiple fractional power series about $t=t_{0}$, where $t$ is a variable and $f_{m}$ are functions of $x$ called the coefficients of the series.

Theorem 3 (see $[11,12])$. Suppose that $u(x, t)$ has a multiple power series representation at $t=t_{0}$ of the form

$$
\begin{aligned}
u(x, t)=\sum_{m=0}^{\infty} f_{m}(x)\left(t-t_{0}\right)^{k \alpha}, \\
\quad 0 \leq n-1<\alpha \leq n, x \in I, t_{0} \leq t<t_{0}+R .
\end{aligned}
$$

If $D_{t}^{m \alpha} u(x, t)$ are continuous on $I \times\left(t_{0}, t_{0}+R\right), m=$ $0,1,2, \ldots$, then coefficients $f_{m}(x)$ of (11) are given as

$$
f_{m}(x)=\frac{D_{t}^{m \alpha} u\left(x, t_{0}\right)}{\Gamma(m \alpha+1)}, \quad m=0,1,2, \ldots
$$

where $D_{t}^{m \alpha}=\partial^{m \alpha} / \partial t^{m \alpha}=\partial^{\alpha} / \partial t^{\alpha} \cdot \partial^{\alpha} / \partial t^{\alpha} \cdots \partial^{\alpha} / \partial t^{\alpha}(m-$ times $)$ and $R=\min _{C \in I} R_{C}$, in which $R_{C}$ is the radius of convergence of the fractional power series $\sum_{m=0}^{\infty} f_{m}(c)\left(t-t_{0}\right)^{m \alpha}$.

Now, the RPSM can be proposed by

$$
u(x, t)=\sum_{n=0}^{\infty} f_{n}(x) \frac{t^{n \alpha}}{\Gamma(1+n \alpha)} .
$$

In order to obtain the approximate value of (13), the form of the $i$ th series of $u(x, t)$ is proposed. Then the truncated series $u_{i}(x, t)$ is defined by

$$
u_{i}(x, t)=\sum_{n=0}^{i} f_{n}(x) \frac{t^{n \alpha}}{\Gamma(1+n \alpha)} .
$$

If $t=0, u(x, 0)=f_{0}(x)$. We define the $i$ th residual function as follows:

$$
\operatorname{Res}_{i}(x, t)=D_{t}^{\alpha} u_{i}-u_{i, x x t}+u_{i, x}+\left(\frac{u_{i}^{2}}{2}\right)_{x}
$$

In order to get $f_{n}(x), n \in N^{*}$, we look for the solution of

$$
D_{t}^{(n-1) \alpha} \operatorname{Res}_{n}(x, 0)=0, \quad n \in N^{*},
$$

where $N^{*}=\{1,2,3, \ldots\}$.

\section{Solution of the Time Fractional BBM-Burger Equation by Residual Power Series Method}

The purpose of this paper is to use modified residual power series method to solve the time fractional BBM-Burger equation. The initial condition of the time fractional BBM-Burger equation is (3), and the exact solution of the time fractional BBM-Burger equation is (4). In this section, we use residual power series method to solve the time fractional BBMBurger equation specifically.

$\operatorname{Res}_{i}(x, t)$ is the $i$ th residual function of (2), which is defined as

$$
\begin{aligned}
\operatorname{Res}_{i}(x, t)= & D_{t}^{\alpha} u_{i}(x, t)-u_{i, x x t}(x, t) \\
& +u_{i, x}(x, t)+\left(\frac{u_{i}^{2}(x, t)}{2}\right)_{x} .
\end{aligned}
$$

Step 1 . For $i=1$, the residual function of the time fractional BBM-Burger equation can be written as

$$
\begin{aligned}
\operatorname{Res}_{1}(x, t)= & D_{t}^{\alpha} u_{1}(x, t)-u_{1, x x t}(x, t) \\
& +u_{1, x}(x, t)+\left(\frac{u_{1}^{2}(x, t)}{2}\right)_{x},
\end{aligned}
$$

where $u_{1}(x, t)$ can be written by (13) as

$$
u_{1}(x, t)=f_{0}(x)+f_{1}(x) \frac{t^{\alpha}}{\Gamma(1+\alpha)} .
$$

Then we get

$$
\begin{aligned}
\operatorname{Res}_{1}(x, t)= & D_{t}^{\alpha} u_{1}(x, t)-u_{1, x x t}(x, t)+u_{1, x}(x, t)+\left(\frac{u_{1}^{2}(x, t)}{2}\right)_{x} \\
= & f_{1}(x)-f_{1}^{\prime \prime}(x) \frac{\alpha t^{\alpha-1}}{\Gamma(1+\alpha)}+f_{0}^{\prime}(x)+f_{1}^{\prime}(x) \frac{t^{\alpha}}{\Gamma(1+\alpha)} \\
& +\left[f_{0}(x)+f_{1}(x) \frac{t^{\alpha}}{\Gamma(1+\alpha)}\right] \\
& *\left[f_{0}^{\prime}(x)+f_{1}^{\prime}(x) \frac{t^{\alpha}}{\Gamma(1+\alpha)}\right] \\
= & f_{1}(x)-\frac{1}{2} \operatorname{sech}^{2}\left(\frac{x}{4}\right) \tanh \left(\frac{x}{4}\right) \\
& -\frac{1}{2}\left[\operatorname{sech}^{2}\left(\frac{x}{4}\right)+\frac{f_{1} x t^{\alpha}}{\Gamma(1+\alpha)}\right] \\
& * \operatorname{sech}^{2}\left(\frac{x}{4}\right) * \tanh \left(\frac{x}{4}\right) .
\end{aligned}
$$

For $t=0$, we have

$$
\left.\operatorname{Res}_{1}(x, t)\right|_{t=0}=f_{1}(x)+f_{0}^{\prime}(x)+f_{0}(x) * f_{0}^{\prime}(x) .
$$

In addition, since

$$
f_{0}(x)=u(x, 0)=\operatorname{sech}^{2}\left(\frac{x}{4}\right),
$$


and

$$
f_{0}^{\prime}(x)=-\frac{1}{2} \operatorname{sech}^{2}\left(\frac{x}{4}\right) \tanh \left(\frac{x}{4}\right)
$$

then according to $\operatorname{Res}_{1}(x, 0)=0$, we have

$$
f_{1}(x)=\frac{1}{2} \operatorname{sech}^{2}\left(\frac{x}{4}\right) \tanh \left(\frac{x}{4}\right)+\frac{1}{2} \operatorname{sech}^{4}\left(\frac{x}{4}\right) \tanh \left(\frac{x}{4}\right) .
$$

Step 2. For $i=2$, the residual function of the time fractional BBM-Burger equation can be written as

$$
\begin{aligned}
\operatorname{Res}_{2}(x, t)= & D_{t}^{\alpha} u_{2}(x, t)-u_{2, x x t}(x, t) \\
& +u_{2, x}(x, t)+\left(\frac{u_{2}^{2}(x, t)}{2}\right)_{x},
\end{aligned}
$$

with the condition

$$
u_{2}(x, t)=f_{0}(x)+f_{1}(x) \frac{t^{\alpha}}{\Gamma(1+\alpha)}+f_{2}(x) \frac{t^{2 \alpha}}{\Gamma(1+2 \alpha)} .
$$

Therefore, we can attain

$$
\begin{aligned}
\operatorname{Res}_{2}(x, t)= & D_{t}^{\alpha} u_{2}(x, t)-u_{2, x x t}(x, t)+u_{2, x}(x, t)+\left(\frac{u_{2}^{2}(x, t)}{2}\right)_{x} \\
= & f_{1}(x)+\frac{f_{2}(x) t^{\alpha}}{\Gamma(1+\alpha)}-f_{1}^{\prime \prime}(x) \frac{\alpha t^{\alpha-1}}{\Gamma(1+\alpha)} \\
& -f_{2}^{\prime \prime}(x) \frac{2 \alpha t^{2 \alpha-1}}{\Gamma(1+2 \alpha)}+f_{0}^{\prime}(x) \\
& +f_{1}^{\prime}(x) \frac{t^{\alpha}}{\Gamma(1+\alpha)}+f_{2}^{\prime}(x) \frac{t^{2 \alpha}}{\Gamma(1+2 \alpha)} \\
& +\left(f_{0}(x)+f_{1}(x) \frac{t^{\alpha}}{\Gamma(1+\alpha)}+f_{2}(x) \frac{t^{2 \alpha}}{\Gamma(1+2 \alpha)}\right) \\
& *\left(f_{0}^{\prime}(x)+f_{1}^{\prime}(x) \frac{t^{\alpha}}{\Gamma(1+\alpha)}+f_{2}^{\prime}(x) \frac{t^{2 \alpha}}{\Gamma(1+2 \alpha)}\right) \\
= & f_{2}(x)-\frac{1}{4} \operatorname{sech}^{2}\left(\frac{x}{4}\right) \tanh ^{2}\left(\frac{x}{4}\right) \\
& +\frac{1}{2} \operatorname{sech}^{2}\left(\frac{x}{4}\right)\left(\frac{1}{4}-\frac{1}{4} \tanh ^{2}\left(\frac{x}{4}\right)\right) \\
& -\frac{1}{2} \operatorname{sech}^{4}\left(\frac{x}{4}\right) \tanh ^{2}\left(\frac{x}{4}\right)+\frac{1}{2} \operatorname{sech}^{4}\left(\frac{x}{4}\right) \\
& +\left(\frac{1}{4}-\frac{1}{4} \tanh ^{2}\left(\frac{x}{4}\right)\right)+\left[\frac{1}{2} \operatorname{sech}^{2}\left(\frac{x}{4}\right) \tanh \left(\frac{x}{4}\right)\right. \\
& +\frac{1}{2} \operatorname{sech}^{4}\left(\frac{x}{4}\right) \tanh ^{\left.\left(\frac{x}{4}\right)+\frac{f_{2}(x) t^{\alpha}}{\Gamma(1+\alpha)}\right]}
\end{aligned}
$$

$$
\begin{aligned}
& *\left[-\frac{1}{2} \operatorname{sech}^{2}\left(\frac{x}{4}\right) \tanh \left(\frac{x}{4}\right)+\frac{t^{\alpha}}{\Gamma(1+\alpha)}\right. \\
& *\left(-\frac{1}{4} \operatorname{sech}^{2}\left(\frac{x}{4}\right) \tanh ^{2}\left(\frac{x}{4}\right)+\frac{1}{2} \operatorname{sech}^{4}\left(\frac{x}{4}\right)\right. \\
& \cdot\left(\frac{1}{4}-\frac{1}{4} \tanh ^{2}\left(\frac{x}{4}\right)\right)-\frac{1}{2} \operatorname{sech}^{4}\left(\frac{x}{4}\right) \tanh ^{2}\left(\frac{x}{4}\right) \\
& \left.\left.+\frac{1}{2} \operatorname{sech}^{4}\left(\frac{x}{4}\right)\left(\frac{1}{4}-\frac{1}{4} \tanh ^{2}\left(\frac{x}{4}\right)\right)\right)\right] \\
& +\left[\operatorname{sech}^{2}\left(\frac{x}{4}\right)+\frac{f_{2}(x) t^{2 \alpha}}{\Gamma(1+2 \alpha)}+\frac{t^{\alpha}}{\Gamma(1+\alpha)}\right. \\
& *\left(\frac{1}{2} \operatorname{sech}^{2}\left(\frac{x}{4}\right) \tanh \left(\frac{x}{4}\right)\right. \\
& \left.+\frac{1}{2} \operatorname{sech}^{4}\left(\frac{x}{4}\right) \tanh \left(\frac{x}{4}\right)\right) \\
& *\left(-\frac{1}{4} \operatorname{sech}^{2}\left(\frac{x}{4}\right) \tanh ^{2}\left(\frac{x}{4}\right)+\frac{1}{2} \operatorname{sech}^{2}\left(\frac{x}{4}\right)\right. \\
& \cdot\left(\frac{1}{4}-\frac{1}{4} \tanh ^{2}\left(\frac{x}{4}\right)\right)-\frac{1}{2} \operatorname{sech}^{4}\left(\frac{x}{4}\right) \tanh ^{2}\left(\frac{x}{4}\right) \\
& \left.\left.+\frac{1}{2} \operatorname{sech}^{4}\left(\frac{x}{4}\right)\left(\frac{1}{4}-\frac{1}{4} \tanh ^{2}\left(\frac{x}{4}\right)\right)\right)\right] .
\end{aligned}
$$

Then we solve $D_{t}^{\alpha} \operatorname{Res}_{2}(x, 0)=0$; thus,

$$
\begin{aligned}
f_{2}(x)= & \frac{7}{8} \operatorname{sech}^{6}\left(\frac{x}{4}\right) \tanh ^{2}\left(\frac{x}{4}\right)-\frac{1}{8} \operatorname{sech}^{6}\left(\frac{x}{4}\right) \\
& +\frac{5}{4} \operatorname{sech}^{4}\left(\frac{x}{4}\right) \tanh ^{2}\left(\frac{x}{4}\right)-\frac{1}{4} \operatorname{sech}^{4}\left(\frac{x}{4}\right) \\
& +\frac{3}{8} \operatorname{sech}^{2}\left(\frac{x}{4}\right) \tanh ^{2}\left(\frac{x}{4}\right)-\frac{1}{8} \operatorname{sech}^{2}\left(\frac{x}{4}\right)
\end{aligned}
$$

Step 3. For $i=3$, the residual function of the time fractional BBM-Burger equation can be written by

$$
\begin{aligned}
\operatorname{Res}_{3}(x, t)= & D_{t}^{\alpha} u_{3}(x, t)-u_{3, x x t}(x, t) \\
& +u_{3, x}(x, t)+\left(\frac{u_{3}^{2}(x, t)}{2}\right)_{x},
\end{aligned}
$$

with the condition

$$
\begin{aligned}
u_{3}(x, t)= & f_{0}(x)+f_{1}(x) \frac{t^{\alpha}}{\Gamma(1+\alpha)} \\
& +f_{2}(x) \frac{t^{2 \alpha}}{\Gamma(1+2 \alpha)}+f_{3}(x) \frac{t^{3 \alpha}}{\Gamma(1+3 \alpha)}
\end{aligned}
$$

Then we can get

$\operatorname{Res}_{3}(x, t)=D_{t}^{\alpha} u_{3}(x, t)-u_{3, x x t}(x, t)+u_{3, x}(x, t)+\left(\frac{u_{3}^{2}(x, t)}{2}\right)_{x}$ 


$$
\begin{aligned}
= & f_{1}(x)+\frac{f_{2}(x) t^{\alpha}}{\Gamma(1+\alpha)}+\frac{f_{3}(x) t^{2 \alpha}}{\Gamma(1+2 \alpha)}-f_{1}^{\prime \prime}(x) \frac{\alpha t^{\alpha-1}}{\Gamma(1+\alpha)} \\
& -f_{2}^{\prime \prime}(x) \frac{2 \alpha t^{2 \alpha-1}}{\Gamma(1+2 \alpha)}-f_{3}^{\prime \prime}(x) \frac{3 \alpha t^{3 \alpha-1}}{\Gamma(1+3 \alpha)}+f_{0}^{\prime}(x) \\
& +f_{1}^{\prime}(x) \frac{t^{\alpha}}{\Gamma(1+\alpha)}+f_{2}^{\prime}(x) \frac{t^{2 \alpha}}{\Gamma(1+2 \alpha)} \\
& +f_{3}^{\prime}(x) \frac{t^{3 \alpha}}{\Gamma(1+3 \alpha)}+\left(f_{0}(x)+f_{1}(x) \frac{t^{\alpha}}{\Gamma(1+\alpha)}\right. \\
& \left.+f_{2}(x) \frac{t^{2 \alpha}}{\Gamma(1+2 \alpha)}+f_{3}(x) \frac{t^{3 \alpha}}{\Gamma(1+3 \alpha)}\right) \\
& *\left(f_{0}^{\prime}(x)+f_{1}^{\prime}(x) \frac{t^{\alpha}}{\Gamma(1+\alpha)}+f_{2}^{\prime}(x) \frac{t^{2 \alpha}}{\Gamma(1+2 \alpha)}\right. \\
& \left.+f_{3}^{\prime}(x) \frac{t^{3 \alpha}}{\Gamma(1+3 \alpha)}\right)
\end{aligned}
$$

So, from $D_{t}^{2 \alpha} \operatorname{Res}_{3}(x, 0)=0$, we get

$$
\begin{aligned}
f_{3}(x)= & \frac{35}{16} \operatorname{sech}^{8}\left(\frac{x}{4}\right) \tanh ^{3}\left(\frac{x}{4}\right)-\frac{11}{16} \operatorname{sech}^{8}\left(\frac{x}{4}\right) \tanh \left(\frac{x}{4}\right) \\
& +\frac{17}{4} \operatorname{sech}^{6}\left(\frac{x}{4}\right) \tanh ^{3}\left(\frac{x}{4}\right)-\frac{13}{8} \operatorname{sech}^{6}\left(\frac{x}{4}\right) \tanh \left(\frac{x}{4}\right) \\
& +\frac{39}{16} \operatorname{sech}^{4}\left(\frac{x}{4}\right) \tanh ^{3}\left(\frac{x}{4}\right)-\frac{19}{16} \operatorname{sech}^{4}\left(\frac{x}{4}\right) \tanh \left(\frac{x}{4}\right) \\
& +\frac{3}{8} \operatorname{sech}^{2}\left(\frac{x}{4}\right) \tanh ^{3}\left(\frac{x}{4}\right)-\frac{1}{4} \operatorname{sech}^{2}\left(\frac{x}{4}\right) \tanh \left(\frac{x}{4}\right) .
\end{aligned}
$$

Step 4. For $i=4$, the residual function of the time fractional BBM-Burger equation can be written as

$$
\begin{aligned}
\operatorname{Res}_{4}(x, t)= & D_{t}^{\alpha} u_{4}(x, t)-u_{4, x x t}(x, t) \\
& +u_{4, x}(x, t)+\left(\frac{u_{4}^{2}(x, t)}{2}\right)_{x},
\end{aligned}
$$

with the condition

$$
\begin{aligned}
u_{4}(x, t)= & f_{0}(x)+f_{1}(x) \frac{t^{\alpha}}{\Gamma(1+\alpha)}+f_{2}(x) \frac{t^{2 \alpha}}{\Gamma(1+2 \alpha)} \\
& +f_{3}(x) \frac{t^{3 \alpha}}{\Gamma(1+3 \alpha)}+f_{4}(x) \frac{t^{4 \alpha}}{\Gamma(1+4 \alpha)} .
\end{aligned}
$$

Using the same method, through the equation of $D_{t}^{3 \alpha}$ $\operatorname{Res}_{4}(x, 0)=0$, we can get $f_{4}(x)$ as follows:

$$
\begin{aligned}
f_{4}(x)= & \frac{385}{64} \operatorname{sech}^{8}\left(\frac{x}{4}\right) \tanh ^{4}\left(\frac{x}{4}\right)-\frac{35}{16} \operatorname{sech}\left(\frac{x}{4}\right) \\
& \cdot\left(-\frac{1}{2} \operatorname{sech}^{2}\left(\frac{x}{4}\right) \tanh \left(\frac{x}{4}\right)\right)^{8} \tanh \left(\frac{x}{4}\right) \\
& *\left(-\frac{1}{2} \operatorname{sech}^{2}\left(\frac{x}{4}\right) \tanh \left(\frac{x}{4}\right)\right)^{3}-\frac{51}{16} \operatorname{sech}^{8}\left(\frac{x}{4}\right) \tanh ^{2}
\end{aligned}
$$

$$
\begin{aligned}
& \cdot\left(\frac{x}{4}\right)+\frac{153}{16} \operatorname{sech}^{6}\left(\frac{x}{4}\right) \tanh ^{4}\left(\frac{x}{4}\right)+\frac{11}{16} \operatorname{sech}\left(\frac{x}{4}\right) \\
& \cdot\left(-\frac{1}{2} \operatorname{sech}^{2}\left(\frac{x}{4}\right) \tanh \left(\frac{x}{4}\right)\right)^{8} \tanh \left(\frac{x}{4}\right) \\
& \cdot\left(-\frac{1}{2} \operatorname{sech}^{2}\left(\frac{x}{4}\right) \tanh \left(\frac{x}{4}\right)\right)-\frac{17}{4} \operatorname{sech}\left(\frac{x}{4}\right) \\
& \left(-\frac{1}{2} \operatorname{sech}^{2}\left(\frac{x}{4}\right) \tanh \left(\frac{x}{4}\right)\right)^{6} \tanh \left(\frac{x}{4}\right) \\
& \cdot\left(-\frac{1}{2} \operatorname{sech}^{2}\left(\frac{x}{4}\right) \tanh \left(\frac{x}{4}\right)\right)^{3}+\frac{11}{64} \operatorname{sech}^{8}\left(\frac{x}{4}\right) \\
& -\frac{193}{32} \operatorname{sech}^{6}\left(\frac{x}{4}\right) \tanh ^{2}\left(\frac{x}{4}\right)+\frac{273}{64} \operatorname{sech}^{4}\left(\frac{x}{4}\right) \tanh ^{4} \\
& \cdot\left(\frac{x}{4}\right)+\frac{13}{8} \operatorname{sech}\left(\frac{x}{4}\right)\left(-\frac{1}{2} \operatorname{sech}^{2}\left(\frac{x}{4}\right)\right)^{6} \tanh \left(\frac{x}{4}\right) \\
& \cdot\left(-\frac{1}{2} \operatorname{sech}^{2}\left(\frac{x}{4}\right) \tanh \left(\frac{x}{4}\right)\right)-\frac{39}{16} \operatorname{sech}\left(\frac{x}{4}\right) \\
& \cdot\left(-\frac{1}{2} \operatorname{sech}^{2}\left(\frac{x}{4}\right) \tanh \left(\frac{x}{4}\right)\right)^{4} \tanh \left(\frac{x}{4}\right) \\
& \cdot\left(-\frac{1}{2} \operatorname{sech}^{2}\left(\frac{x}{4}\right) \tanh \left(\frac{x}{4}\right)\right)^{3}+\frac{13}{32} \operatorname{sech}^{6}\left(\frac{x}{4}\right) \\
& -\frac{53}{16} \operatorname{sech}^{4}\left(\frac{x}{4}\right) \tanh ^{2}\left(\frac{x}{4}\right)+\frac{15}{32} \operatorname{sech}^{2}\left(\frac{x}{4}\right) \tanh ^{4}\left(\frac{x}{4}\right) \\
& +\frac{19}{16} \operatorname{sech}\left(\frac{x}{4}\right)\left(-\frac{1}{2} \operatorname{sech}^{2}\left(\frac{x}{4}\right) \tanh \left(\frac{x}{4}\right)\right)^{4} \tanh \\
& \cdot\left(\frac{x}{4}\right)\left(-\frac{1}{2} \operatorname{sech}^{2}\left(\frac{x}{4}\right) \tanh \left(\frac{x}{4}\right)\right)-\frac{3}{8} \operatorname{sech}\left(\frac{x}{4}\right) \\
& \left(-\frac{1}{2} \operatorname{sech}^{2}\left(\frac{x}{4}\right) \tanh \left(\frac{x}{4}\right)\right)^{2} \tanh \left(\frac{x}{4}\right) \\
& \cdot\left(-\frac{1}{2} \operatorname{sech}^{2}\left(\frac{x}{4}\right) \tanh \left(\frac{x}{4}\right)\right)^{3}+\frac{19}{64} \operatorname{sech}^{4}\left(\frac{x}{4}\right) \\
& -\frac{15}{32} \operatorname{sech}^{2}\left(\frac{x}{4}\right) \tanh ^{2}\left(\frac{x}{4}\right)+\frac{1}{4} \operatorname{sech}\left(\frac{x}{4}\right) \\
& \cdot\left(-\frac{1}{2} \operatorname{sech}^{2}\left(\frac{x}{4}\right) \tanh \left(\frac{x}{4}\right)\right)^{2 *} \tanh \left(\frac{x}{4}\right) \\
& \cdot\left(-\frac{1}{2} \operatorname{sech}^{2}\left(\frac{x}{4}\right) \tanh \left(\frac{x}{4}\right)\right)-\operatorname{sech}\left(\frac{x}{4}\right) \\
& -\frac{35}{8} \operatorname{sech}^{8}\left(\frac{x}{4}\right) \tanh ^{4}\left(\frac{x}{4}\right)+\frac{105}{16} \operatorname{sech}^{8}\left(\frac{x}{4}\right) \tanh ^{2} \\
& \cdot\left(\frac{x}{4}\right)\left(\frac{1}{4}-\frac{1}{4} \tanh ^{2}\left(\frac{x}{4}\right)\right)+\frac{11}{8} \operatorname{sech}^{8}\left(\frac{x}{4}\right) \tanh ^{2} \\
& \cdot\left(\frac{x}{4}\right)-\frac{11}{16} \operatorname{sech}^{8}\left(\frac{x}{4}\right)\left(\frac{1}{4}-\frac{1}{4} \tanh ^{2}\left(\frac{x}{4}\right)\right) \\
& -\frac{51}{8} \operatorname{sech}^{6}\left(\frac{x}{4}\right) \tanh ^{4}\left(\frac{x}{4}\right)+\frac{51}{4} \operatorname{sech}^{6}\left(\frac{x}{4}\right) \tanh ^{2}\left(\frac{x}{4}\right) \\
& \text { * }\left(\frac{1}{4}-\frac{1}{4} \tanh ^{2}\left(\frac{x}{4}\right)\right)+\frac{39}{16} \operatorname{sech}^{6}\left(\frac{x}{4}\right) \tanh ^{2}\left(\frac{x}{4}\right) \\
& -\frac{13}{8} \operatorname{sech}^{6}\left(\frac{x}{4}\right)\left(\frac{1}{4}-\frac{1}{4} \tanh ^{2}\left(\frac{x}{4}\right)\right)
\end{aligned}
$$




$$
\begin{aligned}
& -\frac{39}{16} \operatorname{sech}^{4}\left(\frac{x}{4}\right) \tanh ^{4}\left(\frac{x}{4}\right)+\frac{117}{16} \operatorname{sech}^{4}\left(\frac{x}{4}\right) \tanh ^{2}\left(\frac{x}{4}\right) \\
& \cdot\left(\frac{1}{4}-\frac{1}{4} \tanh ^{2}\left(\frac{x}{4}\right)\right)+\frac{19}{16} \operatorname{sech}^{4}\left(\frac{x}{4}\right) \tanh ^{2}\left(\frac{x}{4}\right) \\
& -\frac{19}{16} \operatorname{sech}^{4}\left(\frac{x}{4}\right)\left(\frac{1}{4}-\frac{1}{4} \tanh ^{2}\left(\frac{x}{4}\right)\right) \\
& -\frac{3}{16} \operatorname{sech}^{2}\left(\frac{x}{4}\right) \tanh ^{4}\left(\frac{x}{4}\right)+\frac{9}{8} \operatorname{sech}^{2}\left(\frac{x}{4}\right) \tanh ^{2}\left(\frac{x}{4}\right) \\
& \cdot\left(\frac{1}{4}-\frac{1}{4} \tanh ^{2}\left(\frac{x}{4}\right)\right)+\frac{1}{8} \operatorname{sech}^{2}\left(\frac{x}{4}\right) \tanh ^{2}\left(\frac{x}{4}\right) \\
& -\frac{1}{4} \operatorname{sech}^{2}\left(\frac{x}{4}\right)\left(\frac{1}{4}-\frac{1}{4} \tanh ^{2}\left(\frac{x}{4}\right)\right)^{2}+\frac{1}{16} \operatorname{sech}^{2}\left(\frac{x}{4}\right) .
\end{aligned}
$$

Thus, the approximate solution of the time fractional BBM-Burger equation is

$$
\begin{aligned}
u_{4}(x, t)= & f_{0}(x)+f_{1}(x) \frac{t^{\alpha}}{\Gamma(1+\alpha)}+f_{2}(x) \frac{t^{2 \alpha}}{\Gamma(1+2 \alpha)} \\
& +f_{3}(x) \frac{t^{3 \alpha}}{\Gamma(1+3 \alpha)}+f_{4}(x) \frac{t^{4 \alpha}}{\Gamma(1+4 \alpha)}
\end{aligned}
$$

where $f_{0}(x)$ is given in the initial condition at (3) and $f_{1}(x), f_{2}(x), f_{3}(x)$, and $f_{4}(x)$ are given at (24)-(35).

\section{Results and Discussion}

In this section, the approximate analytical solution of the time fractional BBM-Burger equation by using residual power series method is calculated. We can compare the exact solution of the BBM-Burger equation with the analytical approximate solution by graphics and charts.

In Figure 1, the approximate solutions and the exact solutions are presented by drawing three-dimensional graphics. Figure 1(a) presents the approximate solution, where $\alpha=$ 0.9 , and Figure 1(b) presents the exact solution at $\alpha=1$.

In Figure 1, when $\alpha$ approaches 1, the approximate solution is close to the exact solution. So, we can conclude that when $\alpha$ approaches 1 , the three-dimensional graphic is accurate and when $\alpha$ approaches 0 , the three-dimensional graphic is inaccurate. In such phenomena, one can say that when alpha approaches 0 , the solution bifurcates or admits chaotic behavior.

In Figure 2, the three-dimensional graphics show the influence of different $\alpha$ on analytical solutions. Figure 2(a) presents approximate solutions when $\alpha=0.25$, and Figure 2(b) presents approximate solutions when $\alpha=0.5$. In Figure 3, the three-dimensional graphics show approximate solutions when $\alpha=0.8$ and $\alpha=1$ (Figures 3(a) and 3(b), respectively).

In Figures 2 and 3, we find that the larger the value of $\alpha$ is, the smoother is the plane. As parameter $\alpha$ increases, the graphics get closer and closer to the exact solution of the graphic.

For any $\alpha \in(0,1]$, the exact value of $\operatorname{Res}(x, t)$ is 0 . The difference between the 4 th approximate solutions and the exact solutions can be shown by the value of $\operatorname{Res}(x, t)$. In Figure 4 , the two-dimensional graphics show the influence of different $\alpha$ and $x$ on the value of $\operatorname{Res}(x, t)$. We can find the impact of different $\alpha$ and $x$ on $\operatorname{Res}_{4}(x, t)$ at $t=0.01$ and $t=0.001$. In Figure 4 , the different colors present different curves. In Figure 4(a), the parameter $t$ is 0.01 ; in Figure 4(b), the parameter $t$ is 0.001 .

As shown in Figure 4, if the values of $\alpha$ and $t$ are fixed, when $|x|>8$, the values of $\operatorname{Res}_{4}(x, t)$ are close to 0 . When the values of $x$ are not in this interval, the values of $\operatorname{Res}_{4}(x, t)$ are not close to 0 . For $x \in(-8,8)$, if the values of $x$ and $t$ are fixed, the value of $\left|\operatorname{Res}_{4}(x, t)\right|$ decreases with an increase in $\alpha$, and if the values of $x$ and $\alpha$ are fixed, the value of $\left|\operatorname{Res}_{4}(x, t)\right|$ increases with a decrease in $t$.

In Figure 5, the two-dimensional graphics show the impact of different $x$ and $\alpha$ on the value of $\left|\operatorname{Res}_{4}(x, t)\right|$. If the value of $x$ is fixed, the relationship of $t$ and $\operatorname{Res}_{4}(x, t)$ is presented in the each panel of Figure 5. Different colors present different $\alpha$.

In Figure 5, we can see that if the values of $\alpha$ and $t$ are fixed, the value of $\left|\operatorname{Res}_{4}(x, t)\right|$ decreases with an increase in constant $x$. If the value of $t$ and $x$ are fixed, the value of $\operatorname{Res}_{4}(x, t) \mid$ decreases with an increase in $\alpha$. If the value of $\alpha$ and $x$ are fixed, the value of $\left|\operatorname{Res}_{4}(x, t)\right|$ decreases with an increase in $t \in(0,0.2)$. As shown in Figures 5(a)-5(d), the value of $\left|\operatorname{Res}_{4}(x, 0)\right|$ is a constant and $\left|\operatorname{Res}_{4}(x, 0)\right| \neq 0$. However, if $t \rightarrow 0$, the value of $\left|\operatorname{Res}_{4}(x, t)\right| \rightarrow 0$, which is because the power series approximate solution of $u_{4}(x, t)$ is a generalized Taylor expansion at $t_{0}=0$. If $t \rightarrow t_{0}$, the precision of the power series approximate solution of $u_{4}(x, t)$ is higher.

Then we compare the exact solution with the approximate solution for the time fractional BBM-Burger equation. The absolute error is

$$
\operatorname{Error}(x, t)=\left|u(x, t)_{\text {exact }}-u(x, t)_{\mathrm{RPSM}}\right| \cdot
$$

In Table 1, we present the exact solutions, the 4 th-term approximate solutions by residual power series method, and the absolute errors. We can find the relationship between $x$ and $t$ and the solutions of $u(x, t)_{\text {exact }}$ and $u(x, t)_{\mathrm{RPSM}}$.

In Table 1, we can conclude that the greater the absolute value of $x$ is, the smaller the $u(x, t)_{\text {exact }}$ and $u_{4}(x, t)_{\mathrm{RPSM}}$ values are. At the same condition of $x$, we can find that the smaller the value of $t$ is, the smaller the absolute errors are. There are two conditions at the same value of $t$-one is that the absolute errors are smallest when the value of $x$ is zero and the other is that the smaller the values of absolute $x$ are, the larger are the values of absolute errors. In general, we can find that the absolute errors with different $x$ and $t$ between the 4 th-term analytical approximate solutions and the exact solutions are within the acceptable range. The range of magnitude of absolute errors is from $10^{-3}$ to $10^{-7}$.

As shown in Table 2, we compare the 4th-term approximate solutions by residual power series method (RPSM) with the 5th-term approximate solutions by fractional homotopy analysis transform method (FHATM) in [31].

By comparing 4th-term approximate solutions by residual power series method (RPSM) with the 5th-term approximate solutions by fractional homotopy analysis transform 


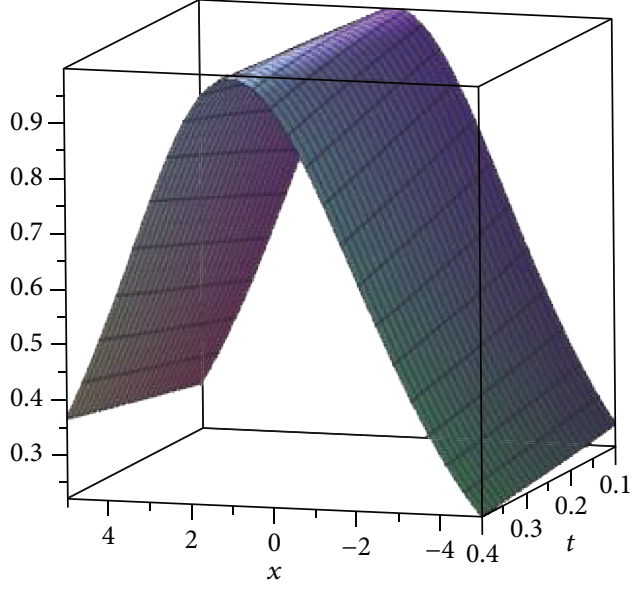

(a) $u_{4}(x, t)_{\mathrm{RPSM}}$ when $\alpha=0.9$

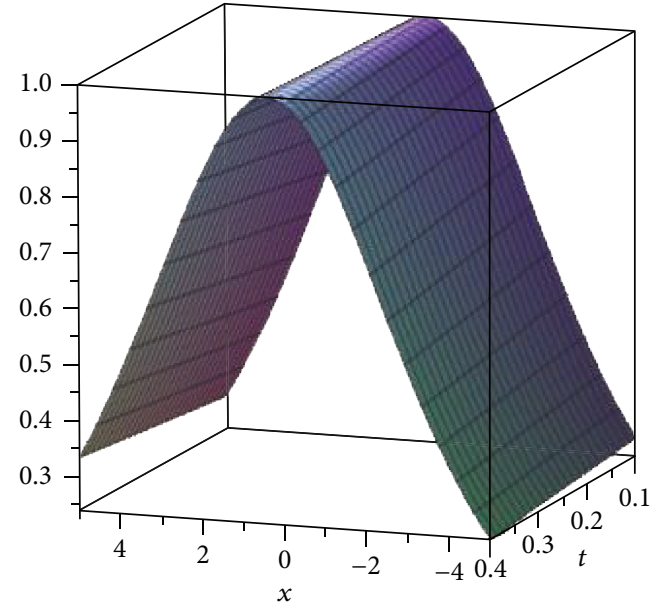

(b) $u(x, t)_{\text {exact }}$ when $\alpha=1$

Figure 1: 3D graphics of the exact and approximate solutions.

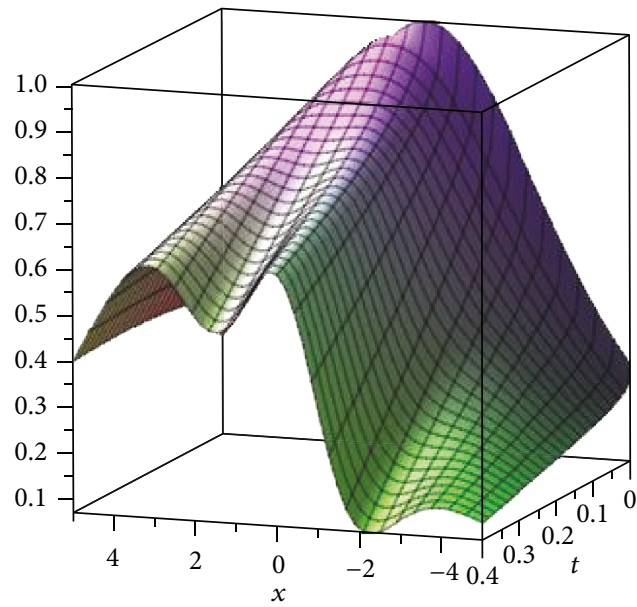

(a) $u_{4}(x, t, \alpha=0.25)$

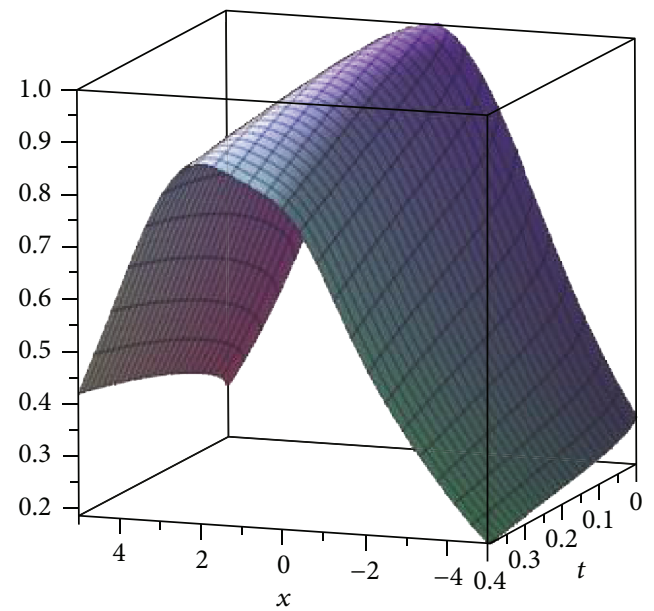

(b) $u_{4}(x, t, \alpha=0.5)$

Figure 2: Approximate solution $u_{4}(x, t, \alpha=0.25,0.5)$.

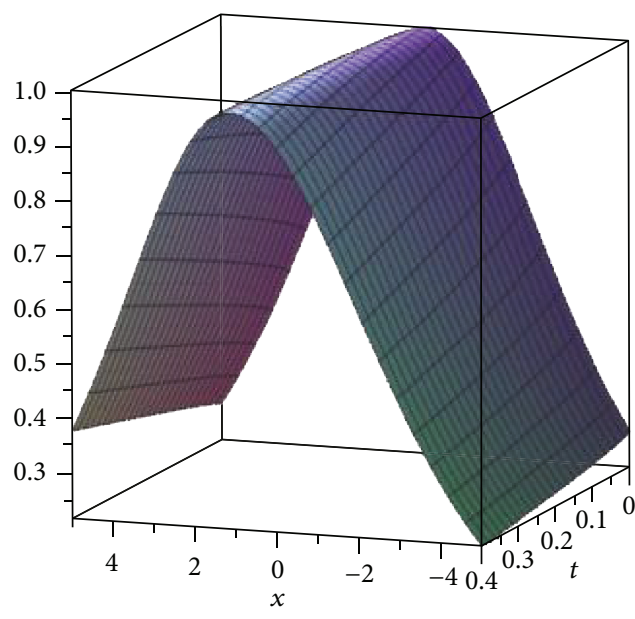

(a) $u_{4}(x, t, \alpha=0.8)$

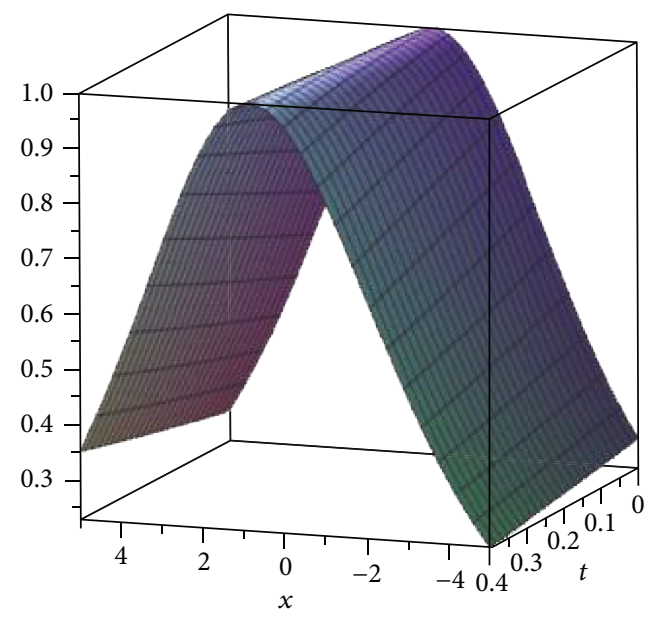

(b) $u_{4}(x, t, \alpha=1)$

FIGURE 3: Approximate solution $u_{4}(x, t, \alpha=0.8,1)$. 


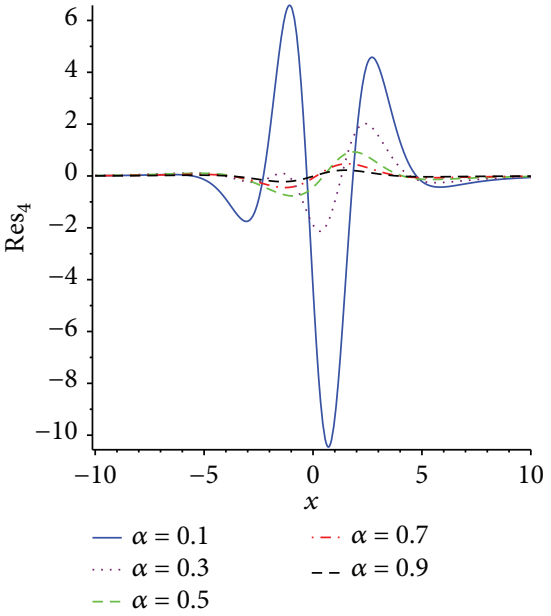

(a) $\operatorname{Res}_{4}(x, t=0.01)$

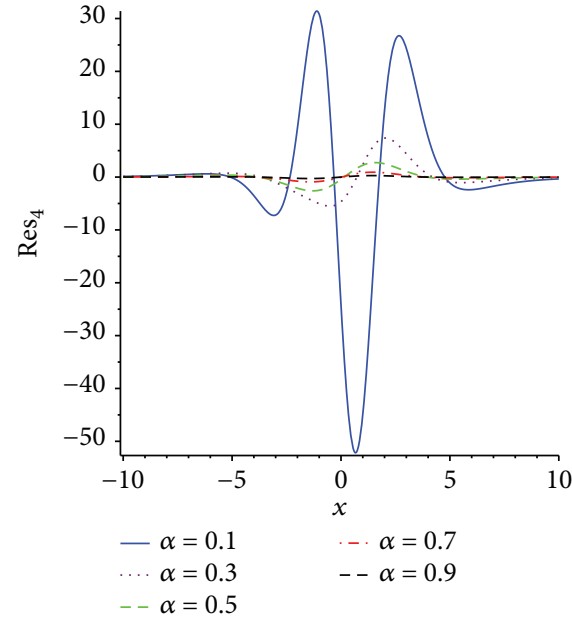

(b) $\operatorname{Res}_{4}(x, t=0.001)$

Figure 4: The impact of different $t$ and $\alpha$ on $\operatorname{Res}_{4}(x, t)$.

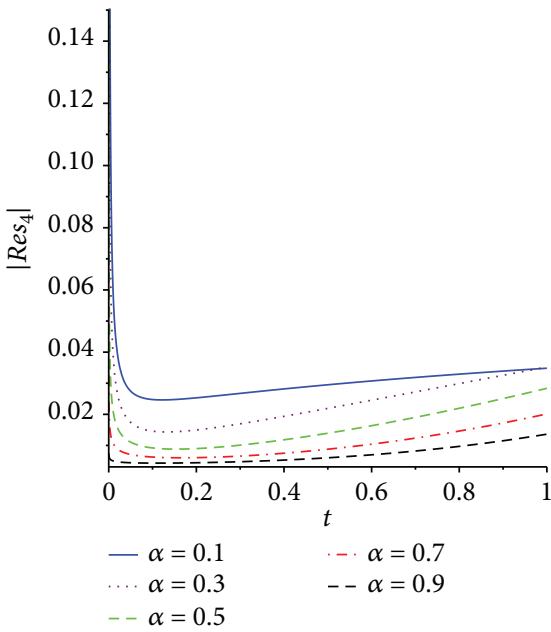

(a) $\left|\operatorname{Res}_{4}(x=10, t)\right|$

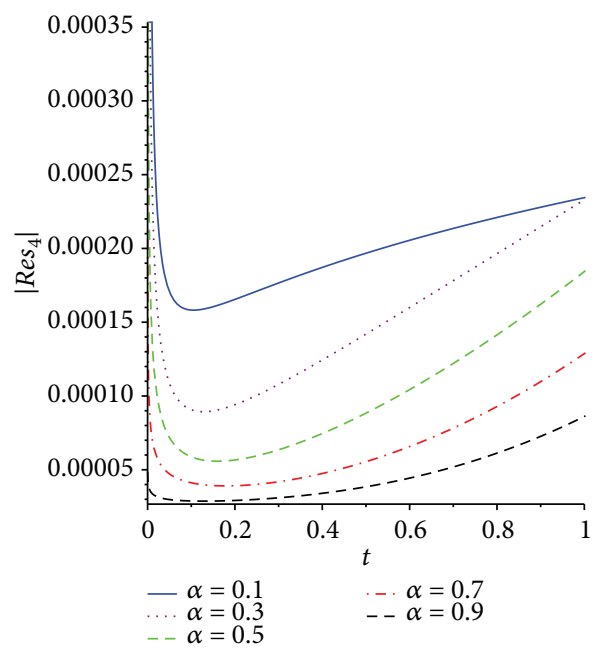

(c) $\left|\operatorname{Res}_{4}(x=20, t)\right|$

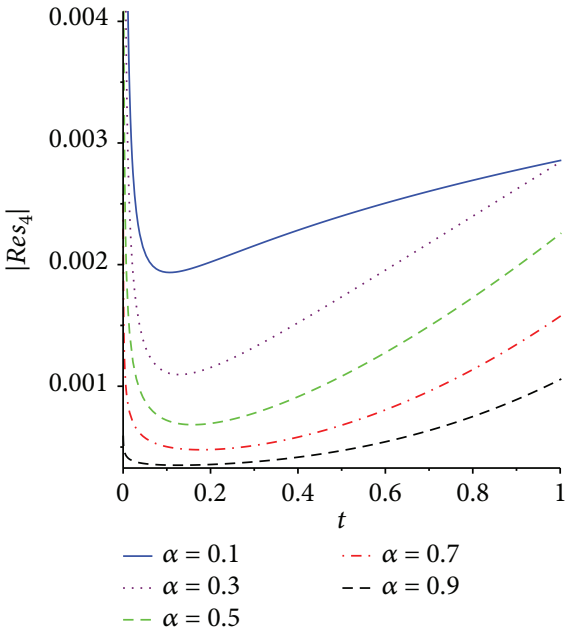

(b) $\left|\operatorname{Res}_{4}(x=15, t)\right|$

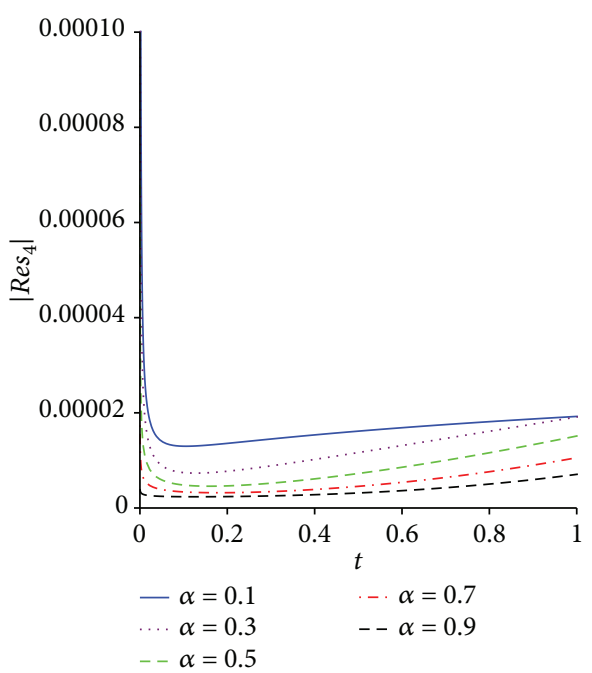

(d) $\mid \operatorname{Res}_{4}(x=25, t)$

Figure 5: The impact of different $t$ and $\alpha$ on $\left|\operatorname{Res}_{4}(x, t)\right|$. 
TABLE 1: Solutions for $\alpha=1$.

\begin{tabular}{|c|c|c|c|c|}
\hline$x$ & $t$ & $u(x, t)_{\text {exact }}$ & $u_{4}(x, t)_{\mathrm{RPSM}}$ & Error $(x, t)_{\mathrm{RPSM}}$ \\
\hline & 0.001 & 0.0022087888 & 0.0022087864 & $2.44 \times 10^{-9}$ \\
\hline \multirow[t]{3}{*}{-15} & 0.01 & 0.0021988825 & 0.0021988584 & $2.41 \times 10^{-8}$ \\
\hline & 0.1 & 0.0021022279 & 0.0021019994 & $2.29 \times 10^{-7}$ \\
\hline & 0.001 & 0.0265791118 & 0.0265787633 & $3.49 \times 10^{-7}$ \\
\hline \multirow[t]{3}{*}{-10} & 0.01 & 0.0264613640 & 0.0264579103 & $3.45 \times 10^{-6}$ \\
\hline & 0.1 & 0.0253118301 & 0.0252801959 & $3.16 \times 10^{-5}$ \\
\hline & 0.001 & 0.2802959508 & 0.2802626306 & $3.33 \times 10^{-5}$ \\
\hline \multirow[t]{3}{*}{-5} & 0.01 & 0.2792275395 & 0.2788971245 & $3.30 \times 10^{-4}$ \\
\hline & 0.1 & 0.2687235583 & 0.2656871698 & $3.30 \times 10^{-3}$ \\
\hline & 0.001 & 0.9999999376 & 0.9999997500 & $1.88 \times 10^{-7}$ \\
\hline \multirow[t]{3}{*}{0} & 0.01 & 0.9999937500 & 0.9999750000 & $1.88 \times 10^{-6}$ \\
\hline & 0.1 & 0.9993752604 & 0.9974997396 & $1.88 \times 10^{-3}$ \\
\hline & 0.001 & 0.2805338222 & 0.2805672046 & $3.34 \times 10^{-5}$ \\
\hline \multirow[t]{3}{*}{5} & 0.01 & 0.2816062545 & 0.2819428891 & $3.37 \times 10^{-4}$ \\
\hline & 0.1 & 0.2925122644 & 0.2961686088 & $3.66 \times 10^{-3}$ \\
\hline & 0.001 & 0.0266053480 & 0.0266056972 & $3.49 \times 10^{-7}$ \\
\hline \multirow[t]{3}{*}{10} & 0.01 & 0.0267237277 & 0.0267272511 & $3.52 \times 10^{-6}$ \\
\hline & 0.1 & 0.0279364632 & 0.0279748560 & $3.84 \times 10^{-5}$ \\
\hline & 0.001 & 0.0022109963 & 0.0022109987 & $2.44 \times 10^{-9}$ \\
\hline \multirow[t]{2}{*}{15} & 0.01 & 0.0022209571 & 0.0022209817 & $2.46 \times 10^{-8}$ \\
\hline & 0.1 & 0.0023230642 & 0.0023233232 & $2.59 \times 10^{-7}$ \\
\hline
\end{tabular}

TABLE 2: Comparison between $\operatorname{Error}(x, t)_{\mathrm{RPSM}}$ and $\operatorname{Error}(x, t)_{\mathrm{FHATM}}^{[31]}$ at $\alpha=1$.

\begin{tabular}{cccccc}
\hline$x$ & $t$ & $u(x, t)_{\mathrm{exact}}$ & $u_{4}(x, t)_{\mathrm{RPSM}}$ & Error $(x, t)_{\mathrm{RPSM}}$ & Error $(x, t)_{\mathrm{FHATM}}^{[31]}$ \\
\hline \multirow{2}{*}{10} & 0.01 & $2.672 \times 10^{-2}$ & $2.673 \times 10^{-2}$ & $3.523 \times 10^{-6}$ & $4.529 \times 10^{-5}$ \\
& 0.001 & $2.661 \times 10^{-2}$ & $2.661 \times 10^{-2}$ & $3.492 \times 10^{-7}$ & $4.501 \times 10^{-6}$ \\
15 & 0.01 & $2.221 \times 10^{-3}$ & $2.221 \times 10^{-3}$ & $2.464 \times 10^{-8}$ & $3.717 \times 10^{-6}$ \\
& 0.001 & $2.211 \times 10^{-3}$ & $2.211 \times 10^{-3}$ & $2.441 \times 10^{-9}$ & $3.697 \times 10^{-7}$ \\
20 & 0.01 & $1.825 \times 10^{-4}$ & $1.825 \times 10^{-4}$ & $1.663 \times 10^{-10}$ & $3.034 \times 10^{-7}$ \\
& 0.001 & $1.817 \times 10^{-4}$ & $1.817 \times 10^{-4}$ & $1.640 \times 10^{-11}$ & $3.018 \times 10^{-8}$ \\
\hline
\end{tabular}

TABLE 3: Comparison the 4th residual function for different $\alpha$ and $x$ at $t=0.01$.

\begin{tabular}{lllll}
\hline$\alpha$ & $\operatorname{Res}_{4}(10,0.01)$ & $\operatorname{Res}_{4}(15,0.01)$ & $\operatorname{Res}_{4}(20,0.01)$ & $\operatorname{Res}_{4}(25,0.01)$ \\
\hline 0.1 & -0.3464731857 & -0.0043621541 & -0.0003541594 & -0.0000290446 \\
0.3 & -0.1648036880 & -0.0030670425 & -0.0002505805 & -0.0000205608 \\
0.5 & -0.0618059110 & -0.0017109445 & -0.0001402342 & -0.0000115097 \\
0.7 & -0.0206388560 & -0.0008737493 & -0.0000716902 & -0.0000058845 \\
0.9 & -0.0062622125 & -0.0004139411 & -0.0000339747 & -0.0000027888 \\
\hline
\end{tabular}

method (FHATM), we can find the absolute errors are smaller than the results in [31]. The absolute error by using RPSM is one order of magnitude smaller than that by using
FHATM. So, residual power series method is efficient and accurate for solving the time fractional BBM-Burger equation. In addition, in Table 2 , we can conclude that when the 
value of parameter $t$ is smaller, the absolute error is smaller and when the value of parameter $x$ is larger, the absolute error is smaller; in contrast, when the value of parameter $\alpha$ approaches 1 , the absolute error is smaller.

We compare the value of the 4 th residual function for different $\alpha$ at $t=0.01$ in Table 3 .

In Table 3, we get the solutions of the 4th residual function $\operatorname{Res}_{4}(x, t)$. If the value of $t$ and $\alpha$ are fixed, the value of $\left|\operatorname{Res}_{4}(x, t)\right|$ decreases with an increase in $x$. If the value of $t$ and $x$ are fixed, the value of $\left|\operatorname{Res}_{4}(x, t)\right|$ decreases with an increase in $\alpha$. So, when the value of $\operatorname{Res}_{4}(x, t)$ is close to 0 , the approximate solutions are close to exact solutions and the approximate solutions are accurate. In Table 3, we can conclude that when the value of $\alpha$ is approaching 1 , the solutions are more accurate.

In conclusion, the residual power series method is a powerful method to solve the analytical approximate solution of the time fractional BBM-Burger equation in $|x|>10$ and $t \in(0,0.2)$.

\section{Conclusion}

In this paper, we discuss the analytical solution of the time fractional BBM-Burger equation by using residual power series method (RPSM). The time fractional BBM-Burger equation is calculated by Maple in Windows 7 (64 bit). The analytical solution is presented by graphics and datum. Results show that the analytical solutions by residual power series method are close to the exact solution. In general, RPSM is an effective and convenient method in finding analytical solution for the time fractional BBM-Burger equation and other long waves in certain nonlinear dispersive systems.

\section{Data Availability}

The data used to support the findings of this study are available from the corresponding author upon request.

\section{Conflicts of Interest}

The authors declare that they have no conflicts of interest.

\section{Acknowledgments}

This work is supported by the National Natural Science Foundation of China (Grant no.11701446, 11601420, 11401469, 60974082, and 61741216), New Star Team of Xi'an University of Posts and Telecommunications, Construction of Special Funds for Key Disciplines in Shaanxi Universities, the Science Plan Foundation of the Education Bureau of Shaanxi Province (no.2013JK 1130), Natural Science Foundation of Shaanxi Province (2018JM1055), and Project of Youth Star in Science and Technology of Shaanxi Province (2016KJXX-95).

\section{References}

[1] A. A. Kilbas, H. M. Srivastava, and J. J. Trujillo, "Theory and applications of fractional differential equations," North-
Holland Mathematics Studies, vol. 204, no. 49-52, pp. $2453-$ 2461, 2006.

[2] A. Arikoglu and I. Ozkol, "Solution of fractional differential equations by using differential transform method," Chaos Solitons and Fractals, vol. 34, no. 5, pp. 1473-1481, 2007.

[3] V. Lakshmikantham and A. S. Vatsala, "Basic theory of fractional differential equations," Nonlinear Analysis Theory Methods and Applications, vol. 69, no. 8, pp. 2677-2682, 2008.

[4] K. Diethelm and N. J. Ford, "Multi-order fractional differential equations and their numerical solution," Applied Mathematics and Computation, vol. 154, no. 3, pp. 621-640, 2004.

[5] Z. M. Odibat and S. Momani, "Application of variational iteration method to nonlinear differential equations of fractional order," International Journal of Nonlinear Sciences and Numerical Simulation, vol. 7, no. 1, pp. 27-34, 2006.

[6] S. Liao, "On the homotopy analysis method for nonlinear problems," Applied Mathematics and Computation, vol. 147, no. 2, pp. 499-513, 2004.

[7] R. K. Pandey, O. P. Singh, and V. K. Baranwal, "An analytic algorithm for the space-time fractional advection-dispersion equation," Computer Physics Communications, vol. 182, no. 5, pp. 1134-1144, 2011.

[8] M. Wang, X. Li, and J. Zhang, “The $\left(G^{\prime} / G\right)$-expansion method and travelling wave solutions of nonlinear evolution equations in mathematical physics," Physics Letters A, vol. 372, no. 4, pp. 417-423, 2008.

[9] C. Bota and B. Căruntu, "Analytic approximate solutions for a class of variable order fractional differential equations using the polynomial least squares method," Fractional Calculus and Applied Analysis, vol. 20, no. 4, pp. 1043-1050, 2017.

[10] S. B. Yuste and L. Acedo, "An explicit finite difference method and a new von Neumann-type stability analysis for fractional diffusion equations," SIAM Journal on Numerical Analysis, vol. 42, no. 5, pp. 1862-1874, 2005.

[11] A. El-Ajou, O. A. Arqub, Z. A. Zhour, and S. Momani, "New results on fractional power series: theories and applications," Entropy, vol. 15, no. 12, pp. 5305-5323, 2013.

[12] A. El-Ajou, O. A. Arqub, and S. Momani, "Approximate analytical solution of the nonlinear fractional $\mathrm{KdV}$-Burgers equation: a new iterative algorithm," Journal of Computational Physics, vol. 293, pp. 81-95, 2014.

[13] K. Moaddy, M. AL-Smadi, and I. Hashim, "A novel representation of the exact solution for differential algebraic equations system using residual power-series method," Discrete Dynamics in Nature and Society, vol. 2015, Article ID 205207, 12 pages, 2015.

[14] H. M. Jaradat, S. Al-Shar, Q. J. A. Khan, M. Alquran, and K. Al-Khaled, "Analytical solution of time-fractional Drinfeld-Sokolov-Wilson system using residual power series method," IAENG International Journal of Applied Mathematics, vol. 46, no. 1, pp. 64-70, 2016.

[15] L. Wang and X. Chen, "Approximate analytical solutions of time fractional Whitham-Broer-Kaup equations by a residual power series method," Entropy, vol. 17, no. 12, pp. 65196533, 2015.

[16] F. Xu, Y. Gao, X. Yang, and H. Zhang, "Construction of fractional power series solutions to fractional Boussinesq equations using residual power series method," Mathematical Problems in Engineering, vol. 2016, Article ID 5492535, 15 pages, 2016. 
[17] A. Kumar, S. Kumar, and S. P. Yan, "Residual power series method for fractional diffusion equations," Fundamenta Informaticae, vol. 151, no. 1-4, pp. 213-230, 2017.

[18] H. Tariq and G. Akram, "Residual power series method for solving time-space-fractional Benney-Lin equation arising in falling film problems," Journal of Applied Mathematics and Computing, vol. 55, no. 1-2, pp. 683-708, 2017.

[19] O. A. Arqub and H. Rashaideh, "Solution of Lane-Emden equation by residual power series method," in ICIT 2013 The 6th International Conference on Information Technology, Amman, Jordan, April 2013.

[20] M. I. Syam, "Analytical solution of the fractional Fredholm integrodifferential equation using the fractional residual power series method," Complexity, vol. 2017, Article ID 4573589, 6 pages, 2017.

[21] W. Li and Y. Pang, "Asymptotic solutions of time-space fractional coupled systems by residual power series method," Discrete Dynamics in Nature and Society, vol. 2017, Article ID 7695924, 10 pages, 2017.

[22] B. A. Mahmood and M. A. Yousif, "A novel analytical solution for the modified Kawahara equation using the residual power series method," Nonlinear Dynamics, vol. 89, no. 2, pp. 1233-1238, 2017.

[23] M. Alquran and I. Jaradat, "A novel scheme for solving Caputo time-fractional nonlinear equations: theory and application," Nonlinear Dynamics, vol. 91, no. 4, pp. 23892395, 2018.

[24] M. Alquran, H. M. Jaradat, and M. I. Syam, "Analytical solution of the time-fractional Phi-4 equation by using modified residual power series method," Nonlinear Dynamics, vol. 90, no. 4, pp. 2525-2529, 2017.

[25] M. Alquran, K. Al-Khaled, and J. Chattopadhyay, "Analytical solutions of fractional population diffusion model: residual power series," Mathematical Sciences, vol. 8, no. 4, pp. 153160, 2015.

[26] M. Alquran, K. Al-Khaled, S. Sivasundaram, and H. M. Jaradat, "Mathematical and numerical study of existence of bifurcations of the generalized fractional Burgers-Huxley equation," Nonlinear Studies, vol. 24, no. 1, pp. 235-244, 2017.

[27] M. Alquran, "Analytical solution of time-fractional twocomponent evolutionary system of order 2 by residual power series method," Journal of Applied Analysis and Computation, vol. 5, no. 4, pp. 589-599, 2015.

[28] C. I. Kondo and C. M. Webler, "The generalized BBM-Burgers equations: convergence results for conservation law with discontinuous flux function," Applicable Analysis, vol. 95, no. 3, pp. 503-523, 2016.

[29] L. N. M. Tawfiq and Z. R. Yahya, "Using cubic trigonometric B-spline method to solve BBM-Burger equation," in MDSG CONFERENCE 2016 Conferences, Sintok, Kedah, March 2016.

[30] M. Shakeel, Q. M. Ul-Hassan, J. Ahmad, and T. Naqvi, "Exact solutions of the time fractional BBM-Burger equation by novel ( $\left.\mathrm{G}^{\prime} / \mathrm{G}\right)$-expansion method," Advances in Mathematical Physics, vol. 2014, Article ID 181594, 15 pages, 2014.

[31] S. Kumar and D. Kumar, "Fractional modelling for BBMBurger equation by using new homotopy analysis transform method," Journal of the Association of Arab Universities for Basic and Applied Sciences, vol. 16, no. 1, pp. 16-20, 2014.
[32] A. Fakhari, G. Domairry, and Ebrahimpour, "Approximate explicit solutions of nonlinear BBMB equations by homotopy analysis method and comparison with the exact solution," Physics Letters A, vol. 368, no. 1-2, pp. 64-68, 2007.

[33] I. Podlubny, "Fractional differential equations: an introduction to fractional derivatives, fractional differential equations, to methods of their solution and some of their applications," Mathematics in Science and Engineering, vol. 198, pp. 1-340, 1999. 


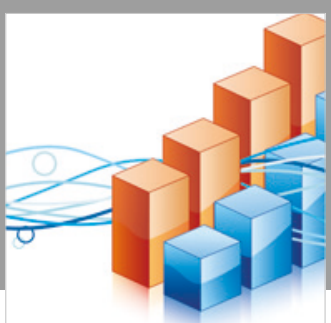

Advances in

Operations Research

\section{-n-m}
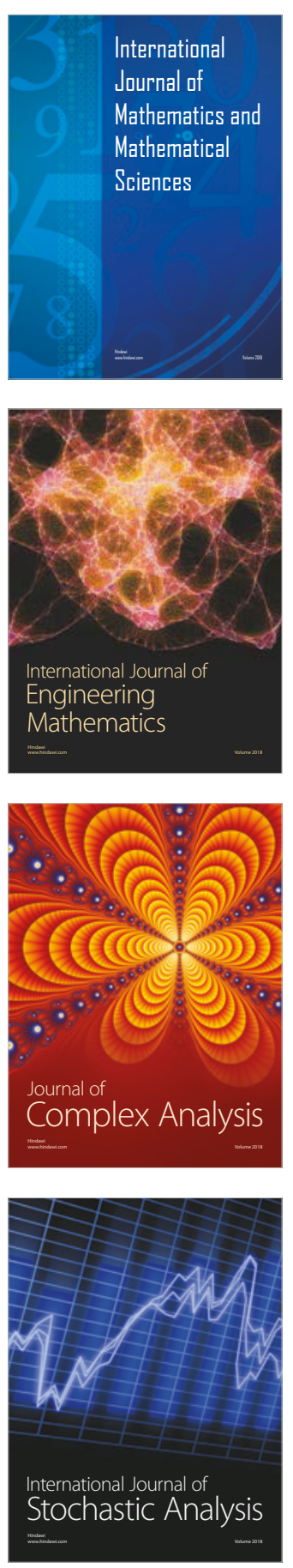
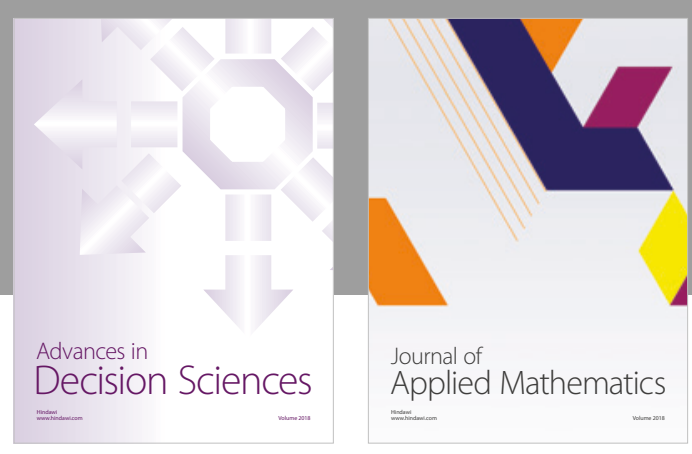

Journal of

Applied Mathematics
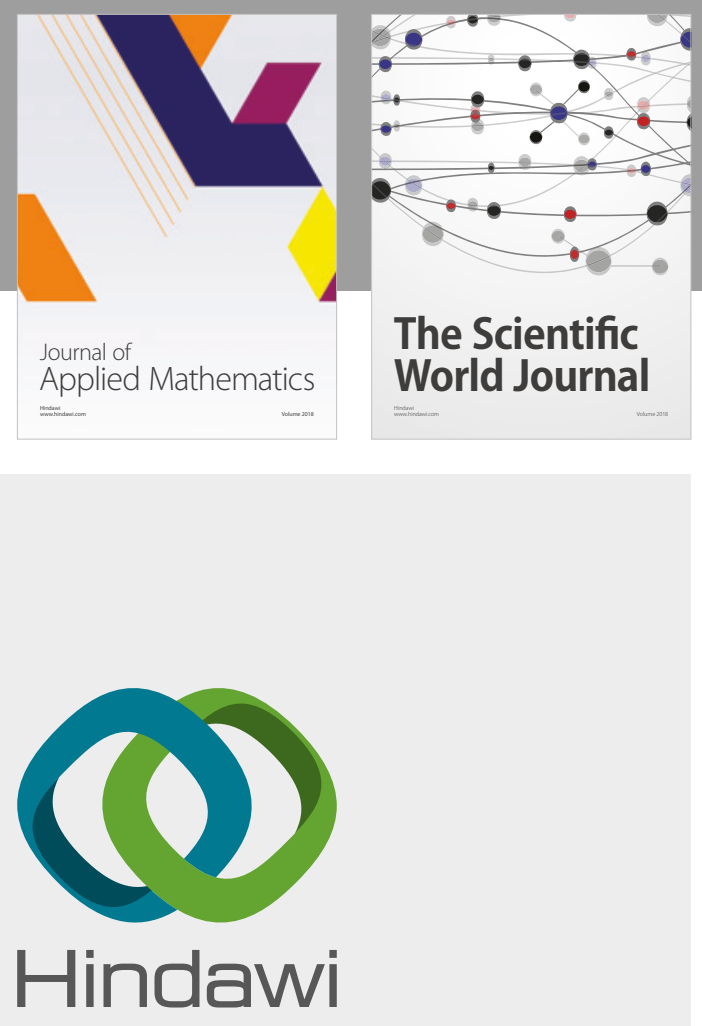

Submit your manuscripts at

www.hindawi.com

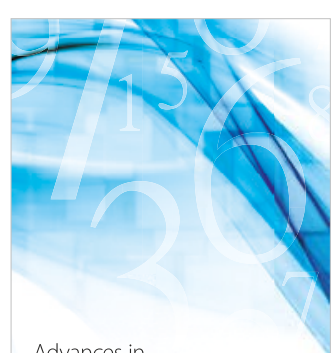

Advances in
Numerical Analysis
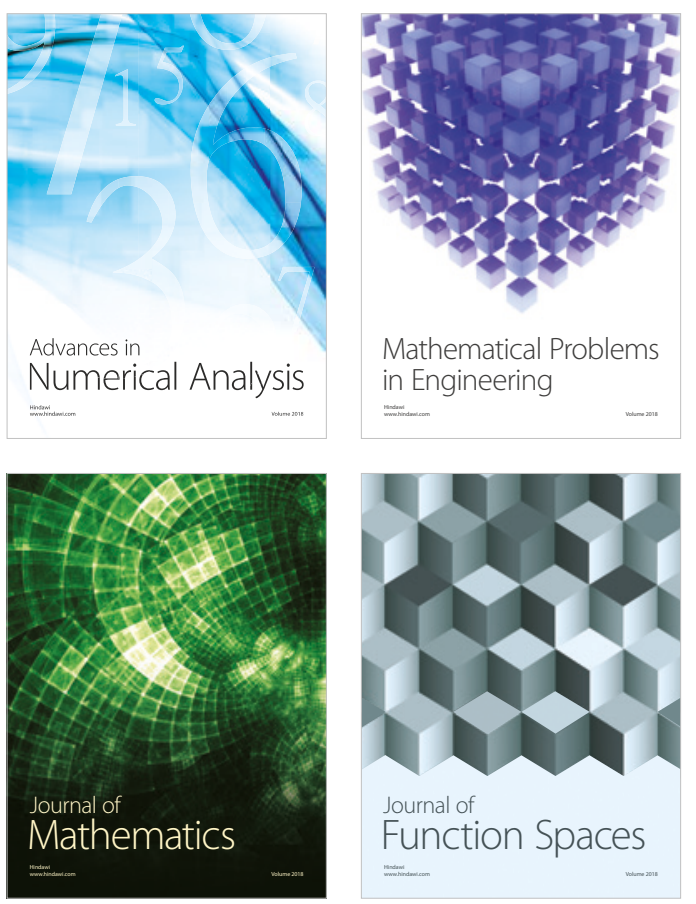

Mathematical Problems in Engineering

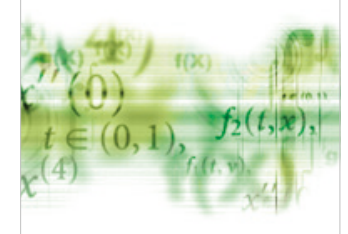

International Journal of

Differential Equations

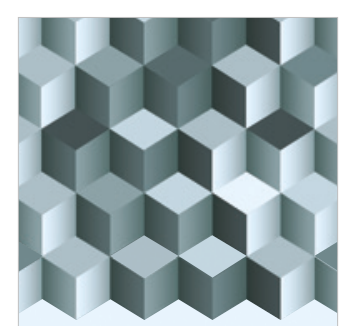

Journal of

Function Spaces
The Scientific

World Journal

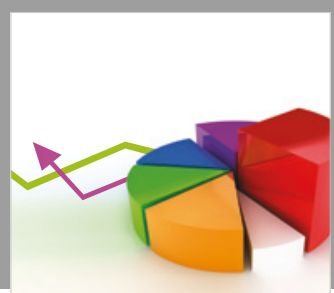

Journal of

Probability and Statistics
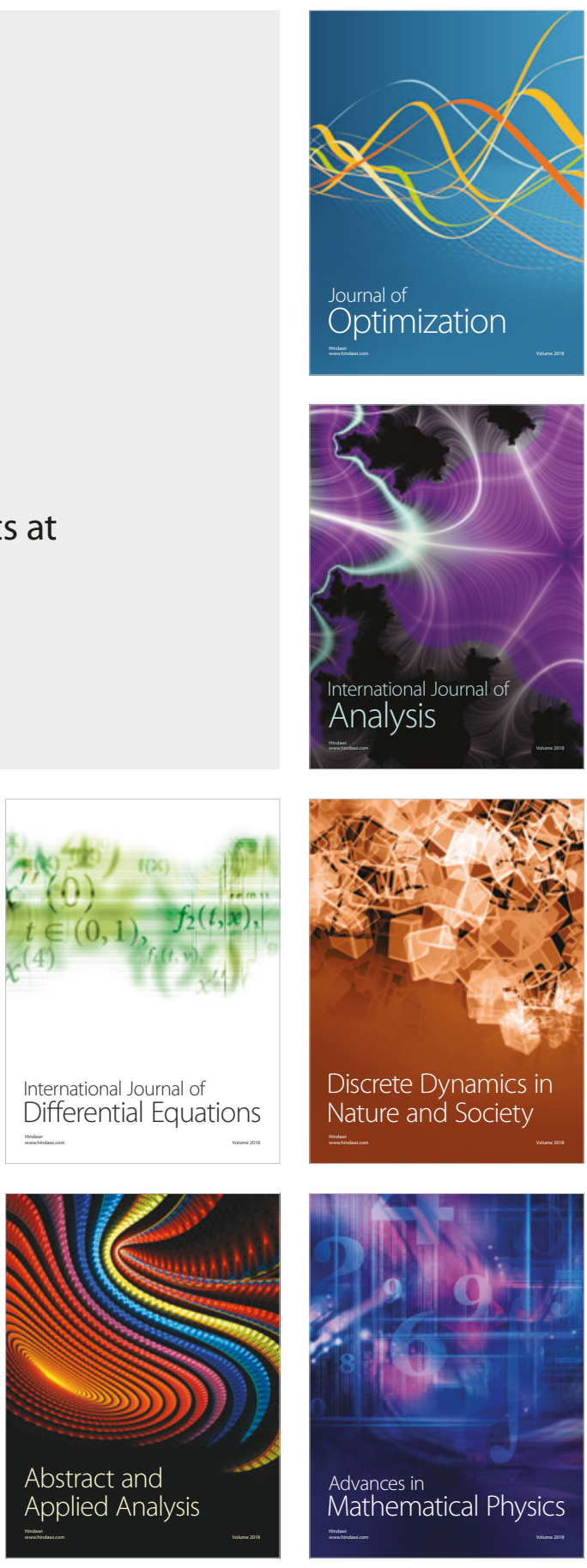